\title{
D-lactic acidosis and ataxia in a man with Crohn disease
}

\author{
Paul D. James MD MSc, David Black MD, Ayelet Kuper MD DPhil, Fred Saibil MD
}

Previously published at www.cmaj.ca

$\mathrm{A}$ 49-year-old man presented to the emergency department with an eight-hour history of unsteady gait, impaired concentration, difficulty speaking and blurry vision, which had developed over the previous seven hours. During the preceding two days, he had been excessively thirsty and drank large amounts of water, cola and a "sports drink." He had eaten a large portion of rice with dinner the evening before presentation. On the day he presented, he had awoken in the morning feeling dizzy. As he was leaving for work, he had experienced difficulty using his keys and turning doorknobs.

The patient's medical history included Crohn disease and two major small-bowel resections at 33 and 35 years of age that had left about one metre of small intestine. He had undergone an esophageal bougienage three weeks earlier for a stricture related to Crohn disease. After the bougienage, the patient had regained his ability to consume solids and dramatically increased his caloric intake to regain weight. His diet consisted mainly of fast food, candy bars and soft drinks.

The patient reported that he did not smoke, consume substantial amounts of alcohol or have any drug allergies. On a

Table 1: Laboratory values on admission

\begin{tabular}{lrc}
\hline Laboratory investigation & Result & $\begin{array}{c}\text { Normal range, } \\
\mathrm{mmol} / \mathrm{L}^{*}\end{array}$ \\
\hline Sodium & 139.0 & $135-147$ \\
Potassium & 4.3 & $3.5-5.0$ \\
\hline Chloride & 107.0 & $95-107$ \\
Carbon dioxide & 10.0 & $22-30$ \\
\hline Random glucose & 7.1 & $4.0-8.0$ \\
Albumin & 39.0 & $35-50 \mathrm{~g} / \mathrm{L}$ \\
\hline Osmolality & 292.0 & $280-300 \mathrm{mmol} / \mathrm{kg}$ \\
\hline Urea & 2.3 & $3.0-7.0$ \\
\hline Arterial pH & 7.21 & $7.35-7.45$ \\
\hline $\begin{array}{l}\text { Arterial partial pressure } \\
\text { of carbon dioxide }\left(\mathrm{pco}_{2} \text { ) }\right.\end{array}$ & 23.0 & $35-45 \mathrm{~mm} \mathrm{Hg}$ \\
\hline $\begin{array}{l}\text { Arterial partial pressure } \\
\text { of oxygen }\left(\mathrm{po}_{2} \text { ) }\right.\end{array}$ & 119.0 & $80-100 \mathrm{~mm} \mathrm{Hg}$ \\
\hline Arterial bicarbonate & 9.0 & $22-26$ \\
\hline
\end{tabular}

*Unless otherwise indicated.

\section{Key points}

- When common causes of mixed, normal or large anion gap metabolic acidosis have been ruled out, a comprehensive review of less common causes should be considered.

- D-lactic acidosis should be considered in patients at risk for short-bowel syndrome who have new-onset gait ataxia, cerebellar dysfunction and metabolic acidosis with a large anion gap.

- Treatment consists mainly of intravenous fluid resuscitation and poorly absorbed oral antibiotics. Prevention strategies include a low-carbohydrate diet, maintenance of hydration and avoidance of simple sugars.

daily basis, he took prednisone $20 \mathrm{mg}$, loperamide $2-4 \mathrm{mg}$, vitamin $\mathrm{B}_{12}$ and multivitamins. Except for the addition of prednisone for the Crohn-related stricture, his regimen of medications had not changed during the past year. He had experienced no changes in his usual Crohn symptoms.

On clinical examination, the patient was alert, oriented and not in distress. His vital signs were normal and he had no significant postural changes. Other than mild vertical and horizontal nystagmus, the results of cranial nerve testing were normal. The patient had truncal ataxia and notable dysmetria on finger-nose testing. He had an unsteady, wide-based gait and could not tolerate walking more than five steps. Rapid alternating movements and heel-shin tests were normal. His strength and reflexes, and the results of sensory testing, were normal. The rest of the clinical examination was unremarkable except for midline abdominal surgical scars.

Initial laboratory investigations are presented in Table 1 and include a serum carbon dioxide level of $10 \mathrm{mmol} / \mathrm{L}$ with an anion gap of $22 \mathrm{mEq} / \mathrm{L}$. Complete blood count, creatinine, coagulation studies, liver enzymes and urinalysis were within normal limits. Arterial blood gas analysis showed a $\mathrm{pH}$ of 7.21, partial pressure of carbon dioxide $\left(\mathrm{pCO}_{2}\right)$ of 23 , partial pressure of oxygen $\left(\mathrm{pO}_{2}\right.$ ) of 119 and $\mathrm{HCO}_{3}^{-}$of 9 . Serum lac-

From the Department of Internal Medicine (James), Faculty of Medicine, University of Toronto; and the Division of Gastroenterology (Black, Saibil, Kuper), Department of Internal Medicine, and Wilson Centre for Research in Education (Kuper), Sunnybrook Health Sciences Centre, University of Toronto, Toronto, Ont.

CMAJ 2010. DOI:10.1503/cmaj.090009 
tates and osmolal gap were normal. Screens for serum salicylates, acetaminophen, alcohol and ketones were negative. A computed tomography scan and magnetic resonance imaging of the patient's brain were also normal.

The patient was admitted for further investigation and given $2 \mathrm{~L}$ of isotonic saline over eight hours for suspected mild hypovolemia. The next morning, his anion gap was normal. The only neurologic symptom that persisted was a sensation of gait imbalance. On re-examination, all abnormal findings had disappeared except for the ataxia, which was markedly improved. Within 24 hours, the patient's symptoms had resolved.

A review of the literature on causes of anion-gap acidosis raised the possibility of D-lactatemia, particularly given the patient's history of multiple small-bowel resections. His first blood sample from the emergency department was refrigerated and sent to a specialized research laboratory for a Dlactate assay.

The patient was discharged with a provisional diagnosis of D-lactic acidosis. He was advised to consume a lowcarbohydrate diet and was given a prescription for metronidazole to be started promptly in the event of recurrence of his neurologic symptoms. This poorly absorbed antibiotic is effective in reducing levels of D-lactate-producing bacteria.

The result of the D-lactate assay came back several days later and was $8 \mathrm{mmol} / \mathrm{L}$ (normal $<1.0 \mathrm{mmol} / \mathrm{L}$ ).

\section{Discussion}

Metabolic acidosis with an elevated anion gap has a limited differential diagnosis (Box 1).,4-6 The laboratory tests investigating these typical causes were negative in our patient. Dlactic acidosis is a much rarer cause of metabolic acidosis with an elevated anion gap. Its presence is suggested by elevation of the anion gap in patients with shortened small bowels when other causes have been excluded (Table 2).

The presence of D-lactic acidosis can be confirmed by a serum assay showing a higher than normal D-lactate level. A level greater than $3 \mathrm{mmol} / \mathrm{L}$ is diagnostic for this condition. Measurement of D-lactate, however, is not available routinely in most hospital laboratories. Urinary D-lactate assays may be faster, easier to use and more sensitive than serologic assays., ${ }^{2,3}$

\section{Association with short-gut syndrome}

Various mechanisms have been proposed to describe the association between D-lactic acidosis and short-gut syndrome. Decreased surface area of the small bowel, as well as inflammation associated with excess bacteria, increase the risk that some carbohydrates may not be absorbed by the small intestine and thus pass undigested into the colon. There, they may serve as substrates for D-lactate-producing bacteria, such as Lactobacillus, Streptococcus, Bifidobacterium and Eubacterium. Growth of these bacteria is favoured in conditions of low luminal $\mathrm{pH}$, which may be enhanced by insufficient neutralization of gastric acid. ${ }^{4}$

The anatomic lesions associated with bowel resections and reductions in bowel motility can provide colonic bacteria with an opportunity to migrate into the small intestine and metabolize carbohydrates before they are absorbed. Excess
Box 1: Differential diagnoses for large and normal anion gap metabolic acidoses ${ }^{1,4-6}$

Common causes of metabolic acidosis with a large anion gap

Production of endogenous acids:

- Ketoacidosis (e.g., associated with diabetes, alcoholism, toxicity, starvation)

- Renal failure

- Lactic acidosis (e.g., caused by poor tissue oxygenation, decreased renal clearance, toxins)

Ingestion of exogenous acids:

- Toxic alcohols (e.g. ethylene glycol and methanol)

- Acetylsalicylic acid

- Paraldehyde

Ingestion of other potential toxins:

- Phenformin

- Iron

- Isoniazid

- Acetaminophen

Common causes of metabolic acidosis with a normal anion gap

Gastrointestinal losses of bicarbonate:

- Diarrhea

- Pancreatic fistula

- High-volume drainage of gastrointestinal tube

Renal losses of bicarbonate:

- Proximal renal tubular acidosis

Impairment of renal hydrogen secretion:

- Distal renal tubular acidosis

- Hypoaldosteronism

Ingestion of potential toxins:

- Toluene

- Carbonic anhydrase inhibitors

- Cholestyramine

Dilutional:

- Rapid infusion of intravenous fluids that do not contain bicarbonate

ingestion of carbohydrates, particularly those that are poorly broken down or absorbed, can then lead to high levels of colonic D-lactic acid. ${ }^{5}$

High-fructose corn syrup has been added increasingly to commercially available sweetened drinks over the past 40 years. Fructose that is joined with glucose in the form of a sucrose disaccharide is well absorbed by glucose transporters. However, because of a lack of fructose-specific intestinal transporters, the absorption of fructose is limited when it is found in mixtures with excess glucose. This limited absorption results in elevated luminal sugar substrates for D-lactic acid production. ${ }^{6}$

Various dietary sources of D-lactate exist, including sour milk, molasses, and certain fruits and vegetables. Clinical investigations into the efficiency with which D-lactate is metabolized have been contradictory. Some studies have found that it is readily metabolized to pyruvate by D-lactate dehydrogenase in the liver and kidneys ${ }^{7}$ at a rate similar to the metabolism of L-lactate. ${ }^{8}$ Other studies have shown that it is metabolized more 
slowly because of lower abundance of specific metabolizing enzymes for D-lactate and slower renal clearance. ${ }^{9}$

\section{Neurologic findings}

The clinical manifestations of D-lactic acidosis are listed in Box $2^{1,4,10}$ and include altered mental status and cerebellar ataxia. The cause of these neurologic abnormalities is debated. A reduction in serum $\mathrm{pH}$ alone is insufficient to explain such manifestations, given that acidosis of comparable severity from other causes does not always result in this presentation. Various theories have been advanced, including suggestions that low $\mathrm{pH}$ may affect intraneural metabolism of L-lactate, which is the preferred substrate of nervous aerobic metabolism, ${ }^{11}$ or may inhibit central and peripheral neurotransmitter production. ${ }^{4,12}$ The accumulation of D-lactate in brain tissue because of naturally low levels of Dlactate dehydrogenase has also been hypothesized. ${ }^{11}$
These theories are not supported by a study showing that infusion of D-lactate into healthy volunteers to a serum concentration of $6 \mathrm{mmol} / \mathrm{L}$ did not cause neurologic symptoms. ${ }^{9}$ This finding suggests the presence of other mediators associated with D-lactate. ${ }^{1,59}$ However, infusing D-lactate into peripheral venous blood may not reproduce the clinical presentation associated with D-lactic acidosis because D-lactate absorbed from the colon passes through the portal circulation and into the liver before being released into the systemic circulation.

\section{Treatment}

Although treatment of D-lactic acidosis includes intravenous fluid resuscitation to promote renal excretion of D-lactic acid, no evidence exists to support this intervention in euvolemic patients. ${ }^{10}$ Poorly absorbed oral antibiotics (e.g., metronidazole, neomycin and vancomycin) are effective in reducing lev-

Table 2: Steps involved in determining the cause of metabolic acidosis

\begin{tabular}{l} 
Step \\
\hline 1. Calculate the anion gap \\
The anion gap is the difference between the \\
measured serum cations (i.e., plasma sodium) and \\
serum anions (i.e., chloride and bicarbonate), \\
or $\mathrm{Na}^{+}-\left(\mathrm{Cl}^{-}+\mathrm{HCO}_{3}^{-}\right)$. \\
The normal anion gap is equal to $12 \pm 2 \mathrm{mEq} / \mathrm{L}$. \\
If the patient has hypoalbuminemia, the result needs \\
to be corrected. The normal anion gap corrected for \\
hypoalbuminemia is equal to the anion gap $+[(44-$ \\
serum albumin $) \times 0.25]$.
\end{tabular}

2. Determine whether the type of acidosis is mixed or non-anion gap

Metabolic acidosis with a normal anion gap is associated with loss of bicarbonate or failure to excrete hydrogen ions from the body (Box 1).

To determine if there is a mixed anion gap or nonanion gap acidosis, compare the difference in the delta bicarbonate to the delta anion gap. A concomitant non-anion gap may be present if (normal $\mathrm{HCO}_{3}^{-}-$measured $\mathrm{HCO}_{3}^{-}$) is greater than (normal anion gap - calculated anion gap).

\section{Determine whether the acidosis is caused by} endogenous acids or ingestion of exogenous acids

A large anion gap metabolic acidosis suggests the addition of exogenous or endogenously generated acids. To determine whether ethanol, methanol or ethylene glycol ingestion may have contributed to an anion gap metabolic acidosis, determine the osmolal gap.

The osmolal gap is equal to (measured osmolality calculated serum osmolality).

Calculated serum osmolality $=2 \times \mathrm{Na}^{+}+$glucose + urea

If the osmolal gap is greater than 10 , ingestion of methanol or ethylene glycol should be considered a possible cause.
Results of testing for arterial blood gas $\left(\mathrm{pH}=7.21, \mathrm{pCO}_{2}=\right.$ 23 and $\mathrm{HCO}_{3}^{-}=9$ ) suggested a metabolic acidosis with respiratory compensation.

Anion gap $=\mathrm{Na}^{+}-\left(\mathrm{Cl}^{-}+\mathrm{HCO}_{3}^{-}\right)=139-(107+9)=23$, which is consistent with metabolic acidosis with a large anion gap.

Delta bicarbonate was $24-10=14$. The delta anion gap was $12 \pm 2-23=-9$ to -13 .

The difference in $\mathrm{HCO}_{3}^{-}$from the expected value was 14 . The difference in the anion gap from the expected value was -9 to -13 . Because only a small discrepancy was observed, a pure large anion gap metabolic acidosis was diagnosed.

The osmolal gap was equal to (measured osmolality calculated osmolality), or $292-(2 \times 139+7.1+2.3)=4.6$, which was not large enough to consider methanol or ethylene glycol ingestion as a cause.

Preliminary screening for the most common causes of a large anion gap metabolic acidosis showed a negative toxicology screen for salicylates and ethanol. Serum lactate, glucose, creatinine and ketones were also normal. Based on clinical suspicion, a serum D-lactate assay was eventually ordered, which was positive. 
Box 2: Clinical manifestations of D-lactic acidosis $\mathbf{s}^{1,4,10}$

Altered mental status

- Disorientation

- Decreased level of consciousness

- Irritability

- Coma

Cerebellar signs

- Ataxia

- Dysarthria

- Gait disturbance

- Impaired motor coordination

- Nystagmus

Weakness

Blurred vision

Psychosis

els of D-lactate-producing bacteria. ${ }^{10}$ Use of sodium bicarbonate to treat acidemia has not been consistently shown effective, and has adverse effects (e.g., volume overload, alkalemia, hypernatremia) that should be considered before it is administered. ${ }^{13,14}$ In a patient with a critical level of D-lactate that may be life-threatening, acute administration of sodium bicarbonate followed by hemodialysis may be indicated. ${ }^{15}$

With treatment, the symptoms associated with D-lactic acidosis tend to have a limited course and typically resolve within 72 hours of onset, possibly owing to efficient hepatic metabolism and renal excretion. ${ }^{4,8}$

\section{Prevention}

Prevention of D-lactic acidosis begins with reduced consumption of substrates that promote the proliferation of acidresistant colonic bacteria in patients with shortened small bowels. Therefore, a diet low in carbohydrates, including avoidance of sweetened beverages, is important. Immediate treatment with antibiotics when early symptoms (e.g., disorientation and dysarthria) are detected can avoid progression to the full spectrum of neurologic abnormalities. ${ }^{4,12}$

Competing interests: None declared.

Acknowledgement: The authors thank Dr. Mitchel Halperin for his helpful insight.

\section{REFERENCES}

1. Uchida H, Yamamoto H, Kisaki Y, et al. D-lactic acidosis in short-bowel syndrome managed with antibiotics and probiotics. J Pediatr Surg 2004;39:634-6.

2. Haschke-Becher E, Baumgartner M, Bachmann C. Assay of D-lactate in urine of infants and children with reference values taking into account data below detection limit. Clin Chim Acta 2000;298:99-109.

3. Inoue Y, Shinka T, Ohse M, et al. Changes in urinary level and configuration ratio of D-lactic acid in patients with short bowel syndrome. J Chromatogr B Analyt Technol Biomed Life Sci 2007;855:109-14.

4. Bakhru MR, Kumar A, Aneja A. A 58-year-old woman with mental status changes. Cleve Clin J Med 2007;74:457-62.

5. Halperin ML, Kamel KS. D-lactic acidosis: turning sugar into acids in the gastrointestinal tract. Kidney Int 1996;49:1-8.

6. Davids MR, Segal AS, Brunengraber $\mathrm{H}$, et al. An unusual cause for ketoacidosis. OJM 2004;97:365-76.

7. Zhang DL, Jiang ZW, Jiang J, et al. D-lactic acidosis secondary to short bowel syndrome. Postgrad Med J 2003;79:110-2.

8. Fine A. Metabolism of D-lactate in the dog and in man. Perit Dial Int 1989;9:99-101

9. Oh MS, Uribarri J, Alveranga D, et al. Metabolic utilization and renal handling of D-lactate in men. Metabolism 1985;34:621-5.

10. Uribarri J, Oh MS, Carroll HJ. D-lactic acidosis. A review of clinical presentation, biochemical features, and pathophysiologic mechanisms. Medicine (Baltimore) 1998;77:73-82.

11. Abeysekara S, Naylor JM, Wassef AW, et al. D-lactic acid-induced neurotoxicity in a calf model. Am J Physiol Endocrinol Metab 2007;293:E558-65.

12. Kaufmann P, Shungu DC, Sano MC, et al. Cerebral lactic acidosis correlates with neurological impairment in MELAS. Neurology 2004;62:1297-302.

13. Stacpoole PW. Lactic acidosis: the case against bicarbonate therapy. Ann Intern Med 1986;105:276-9.

14. Kreisberg RA. Pathogenesis and management of lactic acidosis. Annu Rev Med 1984;35:181-93

15. Luft FC. Lactic acidosis update for critical care clinicians. J Am Soc Nephrol 2001;12(Suppl 17):S15-9.

Correspondence to: Dr. Paul D. James, Faculty of Medicine,

University of Toronto, 190 Elizabeth St., Toronto ON M5G 2C4;

paul.james@utoronto.ca

The section Cases presents brief case reports that convey clear, practical lessons. Preference is given to common presentations of important rare conditions, and important unusual presentations of common problems. Articles start with a case presentation (500 words maximum), and a discussion of the underlying condition follows (1000 words maximum). Generally, up to five references are permitted and visual elements (e.g., tables of the differential diagnosis, clinical features or diagnostic approach) are encouraged. Written consent from patients for publication of their story is a necessity and should accompany submissions. See information for authors at www.cmaj.ca. 